CASE REPORT

\section{Impact of Perturbation-Based Balance Training on Functional Outcomes in a Patient with Chronic Incomplete Spinal Cord Injury: A Case Report}

\author{
Taylor Drake $^{1 *}$ and J.J. Mowder-Tinney ${ }^{1}$ \\ ${ }^{1}$ Department of Physical Therapy, Nazareth College, Rochester, New York, USA
}

\begin{abstract}
Individuals with chronic incomplete spinal cord injury $(\mathrm{SCl})$ are at an increased risk for falls due to impaired neural pathways, thus leading to a decreased ability to initiate a protective stepping reaction to prevent falls. This case report demonstrated the use of an 8-week, task-specific, perturbation-based balance training (PBT) program and assessed the impact on functional outcome measures in a 58-year old male 18 months post incomplete $\mathrm{SCl}$ as a result of surgical tumor removal. Interventions within this program were designed to be salient and task-specific while providing optimal challenge in a safe and controlled environment. Therefore, activities in this program did not require the use of complex laboratory equipment and instead focused on intervention strategies and outcomes that could be utilized with any clinician in a variety of settings. Improvements in all outcome measures, decreased fall risk and number of falls, and improved ability to take a protective stepping reaction were noted after conclusion of the program. These results suggest that perturbation-based balance training within the framework of functional and salient interventions can lead to improved outcomes in those with chronic incomplete SCI. Future work should continue to investigate the effects of PBT with task-specific interventions within this population as well as its impact on standardized outcome measures.
\end{abstract}

\section{G Open Access}

Citation: Drake T, Mowder-Tinney JJ. Impact of Perturbation-Based Balance Training on Functional Outcomes in a Patient with Chronic Incomplete Spinal Cord Injury: A Case Report. Physical Medicine and Rehabilitation. 2017; 1(1):2.

Received: June 25, 2017

Accepted: October 23, 2017

Published: October 31, 2017

\section{Copyright: (c) 2017 Drake et al. This is} an open access article distributed under the terms of the Creative Commons Attribution License, which permits unrestricted use, distribution, and reproduction in any medium, provided the original author and source are credited.

\section{Corresponding author:}

Taylor Drake, PT, DPT,

Department of Physical Therapy,

Nazareth College, Rochester, NY, USA

E-mail: tdrake2@mail.naz.edu

\section{Keywords}

Perturbation-based balance, reactive balance, incomplete spinal cord injury, task-specific.

\section{Introduction}

There are 282,000 people with a spinal cord injury (SCI) living in the United States and approximately 17,000 new cases occur each year. ${ }^{1}$ Approximately half of all individuals with a $\mathrm{SCl}$ have an incomplete lesion. ${ }^{2}$ Functional improvements after an incomplete $\mathrm{SCl}$ vary and can be especially challenging for those with chronic injuries. ${ }^{3}$ Discovering a means to facilitate and expedite recovery in this chronic population is critical given the population's risk for falls. ${ }^{2,4}$

Falls are a significant problem among ambulatory patients with incomplete $\mathrm{SCl}$, with approximately $75 \%$ suffering a fall within one year. ${ }^{4}$ Additionally, $45 \%$ of those with incomplete $\mathrm{SCl}$ reported that falls restricted their ability to engage in the community and participate in productive activity. ${ }^{4}$ While ankle and hip strategies have been found to be effective ways to maintain balance and prevent falls following perturbations, a stepping strategy, or reaction, has also been found to be successful and even the preferred strategy. ${ }^{5}$ Horak, et $a l^{5}$ defined a stepping reaction as an "asymmetrical loading and unloading of the legs to move the base of support under the falling center of mass" and Mansfield, et a/ further stated that in order to prevent falls, this motion must occur quicker than a typical voluntary movement. A protective stepping reaction is a necessary reactive balance response to prevent falls resulting from postural perturbations, ${ }^{5-9}$ and therefore people with an ineffective stepping reaction are likely at an increased risk for falls. ${ }^{6}$

An effective stepping reaction can be improved with specific training, and this training is 
associated with fewer number of falls. ${ }^{6,10-13}$ Perturbation-based balance training (PBT) is defined as "a novel balance training intervention that incorporates exposure to repeated postural perturbations to evoke a rapid balance reaction, enabling the individual to improve control of these reactions with practice." ${ }^{6}$ Studies have shown that intervention programs consisting of PBT principles can improve rapid balance reactions by evoking the body's natural protective reactions, instead of trying to retrain these reactions as voluntary movements. ${ }^{6,14}$ Numerous studies have investigated the effects of retraining reactive balance in both healthy older adults $\mathrm{s}^{6,11,13,15}$ and young adults ${ }^{12,13,16}$ as well as with those with neurological deficits including people with stroke ${ }^{7-10}$ and Parkinson's Disease, ${ }^{6,17}$ showing decreases in number of falls, fall risk, and number of steps required to self-correct. However, research in this area in the incomplete $\mathrm{SCI}$ population is lacking. Various studies in the incomplete $\mathrm{SCl}$ population have used PBT theories as part of their intervention programs, but utilized different kinematic and electromyography results as opposed to standardized outcome measures. ${ }^{18,19}$ Other studies in this population have used standardized outcome measures as well as activity-based and task-specific training, but focused on loco-motor training rather than PBT. ${ }^{20,21}$ Activity-based rehabilitation interventions have been found to be successful in those with neurologic injury, ${ }^{20,22}$ but to the author's knowledge, no studies have investigated the effects of task-specific, activity-based PBT on functional outcomes in individuals with incomplete SCI.

In addition to the gap in evidence for those with incomplete $\mathrm{SCl}$, there is also a gap in studies that utilized realistic equipment to measure and implement reactive balance strategies. Reactive balance interventions have been performed through several different methods in the literature, including repeated slip exposures on sliding platforms with a safety harness during sit to stands ${ }^{13}$ and during gait ${ }^{12}$ as well as repeated motor-driven lateral waist pulls on a force platform. ${ }^{11}$ All of these studies found that this type of training lowered fall risk, but they also all included sophisticated laboratory equipment that is not accessible or practical for most clinicians. This reason, along with the fact that many studies utilized kinematics and spatiotemporal characteristics as outcomes rather than standardized, functional, outcome measures, support previous research that has determined that effective balance reactions are not regularly measured or utilized in interventions by physical therapists. ${ }^{23}$

The purpose of this case report was to demonstrate the use, and assess the impact, of functionally focused interventions that utilized challenging PBT on balance and functional outcomes in people with incomplete SCI.

\section{Materials and Methods}

\section{Case Description}

The client was a 58-year-old male presenting to a Physical Therapy (PT) program's probono outpatient clinic, 18 months status post incomplete $\mathrm{SCl}$ as a result of mid-thoracic level tumor surgical removal.

Prior to the tumor discovery, the client had no significant past medical history, owned his own company, and was very active, including golfing and fishing for leisure. The client resided with his wife and was independent with all aspects of his life.

One month prior to discovery of the spinal cord tumor, the client began to complain of decreased sensation distal to the knee. He went to his primary care physician who ordered blood work and a chest $\mathrm{x}$-ray, with no remarkable findings. The client was referred to a neurosurgeon, but subsequently broke his tibia during ambulation prior to this appointment. While in the emergency room, an MRI was ordered and a tumor was found in the client's spinal cord at the mid-thoracic level. Surgery was the recommended course of treatment.

After the spinal cord tumor resection, additional tumors were then discovered in the client's brain and a lymph node in his trachea. He was also diagnosed with malignant small cell lung cancer at this time. He underwent radiation therapy for the newly diagnosed tumors and lung cancer for 10 days while recovering from the spinal cord resection surgery. Approximately one month later, he underwent an additional round of radiation for the remnants of the spinal tumor. During this time, the client felt unable to independently participate in his leisure activities, work, and some activities of daily living after his surgery due to balance deficits and frequent falling.

Physical therapy included homecare for one month after surgery, at which time he was non- 
ambulatory. He then presented to outpatient therapy, where he had been attending twice weekly for 16 months. The main focus of interventions at this original outpatient clinic was balance training, gait training with a rollator walker and progressing to a cane, and core/hip strengthening to help with balance due to decreased lower extremity (LE) sensation and strength. After 16 months of this type of training and upon presenting to the new clinic, the client continued to suffer approximately 2-3 falls per week. He decreased his attendance to his original outpatient clinic to once weekly, but continued with the same focus, once he began attending the PT program's outpatient clinic also once weekly.

The client's functional status upon presenting to the PT program's clinic included having the ability to independently ambulate with a straight cane (with a rollator walker for longer community distance ambulation) and an articulating hinge ankle-foot orthotic with dorsiflexion assist on his right LE. He had been using primarily the cane instead of the rollator for approximately 6 months prior to presenting to the new clinic. The client was able to independently perform activities of daily living, but was aware that he required increased time to ensure safety and prevent falls. His 2-3 falls per week occurred while doing activities such as sit to stand transfers and actions requiring the displacement of an external load, such as shoveling snow. The client's medication list at this time included hydrocodone and gabapentin as needed for lower extremity pain as well as daily Xaerlto to prevent blood clots due to lower extremity edema. He has remained cancer-free since completing radiation therapy 16 months prior to initiating therapy at the new clinic.

The client's primary goal was to maximize his current level in regards to balance, decrease his fear of falling, decrease his number of falls per week, as well as to strengthen his core and LE as he felt these deficits considerably contributed to his impaired balance abilities.

At the time of initial evaluation, the client demonstrated unimpaired light touch in bilateral upper extremities and proximal to the knee in bilateral LE. He exhibited significant hypoesthesia between the knee and malleoli in bilateral LE with no sensation distal to the malleoli, including both the plantar and dorsal aspect of the ankle. His LE proprioception was also absent in both great toes and ankles with sustained clonus noted in his right ankle. The client's manual muscle test measurements were as follows: hip extensors $3 / 5$ left, $2 / 5$ right; hip flexors 4+/5 left, 4/5 right; hip abductors 4-/5 left, 3+/5 right; ankle plantarflexors: 2/5 left, $2 / 5$ right; ankle dorsiflexors: $3+/ 5$ left, $2+/ 5$ right. He ambulated with a straight cane with a wide base of support, lateral trunk lean to stance side, and increased bilateral hip flexion in terminal stance similar to a steppage gait. The client had two losses of balance during the initial evaluation, occurring when asked to come to an abrupt stop and during turning. He showed no ability to take a protective step and required minimal-moderate assistance to maintain balance. His scores in the Timed Up and Go, Five Timed Sit to Stand, Dynamic Gait Index, and Activities Specific Balance Confidence Scale all placed the client at an increased risk for falls and his distance in the Six Minute Walk Test revealed decreased aerobic capacity.

\section{Outcome Measures}

The following self-reported as well as standardized, functional outcome measures were utilized with this client:

1) Dynamic Gait Index (DGI): This test evaluates functional stability during gait activities and evaluates fall risk. The DGI includes items such as walking with speed changes, head turns, pivot turns, over and around obstacles, and stair climbing. ${ }^{24}$

2) Five Times Sit to Stand (5TSS): This test measures LE strength by evaluating the time taken to complete five repetitions of the sit-to-stand action from a standard height chair with no use of hands. ${ }^{25}$

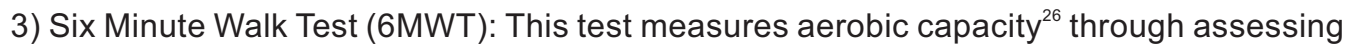
the distance a patient is able to walk over 6 minutes. ${ }^{27}$

4) Activities-Specific Balance Confidence Scale (ABC Scale): This is a 16-item self-report measure in which patients rate their balance confidence for performing certain activities. $^{28}$

5) Timed Up and Go (TUG): This test requires an individual to rise from a chair, walk three meters, and return to the chair to assess walking function, upright mobility, and balance. $^{29,30}$

6) Number of falls per week 


\section{Clinical Impression}

In summary, this client had progressed from using a rollator walker to a straight cane for most daily ambulation approximately 6 months before initiating therapy at the new clinic and was able to independently perform activities of daily living. However, he continued to consistently fall 2-3 times per week, was at an increased risk for falls per standardized outcome measures, and showed little to no ability to take a protective step to prevent falls. From clinical examination and evaluation, it was regarded that the client's impaired sensation, strength, and therefore reactive balance abilities contributed to these impairments.

Due to the frequency and nature of the client's falls, the facilitation and retraining of reactive balance strategies to help his ability to self-correct to prevent falls during everyday, functional activities, was chosen as a focus for intervention Additionally, a heavy emphasis of training focused on a task-specific program encompassing salient activities to help the client improve his ability to maintain balance when unexpectedly perturbed, decrease his fall risk, and decrease fear of falling. The overall aim was to improve the client's ability to take a protective step without hesitation to prevent loss of balance during both activities of daily living as well as leisure activities.

\section{Intervention}

The client was seen for 8 weeks of intervention once weekly, each lasting 60 minutes. Approximately $70 \%$ of interventions were focused on $\mathrm{PBT}$, or retraining reactive balance strategies, and $30 \%$ on outcome measure retesting and functional tasks. The client also continued to attend his original outpatient PT clinic once weekly focused only on general balance/gait training and core/hip stability. Interventions at the new PT program clinic focused on challenging, realistic, purposeful tasks that were meaningful to the client and the design emphasized facilitating and retraining reactive balance to improve ability to self-correct to prevent and decrease falls. Primary interventions included:

1. Facilitation of lateral protective step with a resistance band (Figure 1). The client stood in a corner to increase his confidence and safety, with a resistance band around his waist and held laterally by the clinician. He was initially cued to lean his body into the band and was given a countdown of when the band would be released. The release caused the client to take a protective step to prevent a fall and the countdown helped him anticipate when he needed to step. Once the client had successfully completed this task and was able to take one compensatory step, the countdown was eliminated and the band was released without warning.

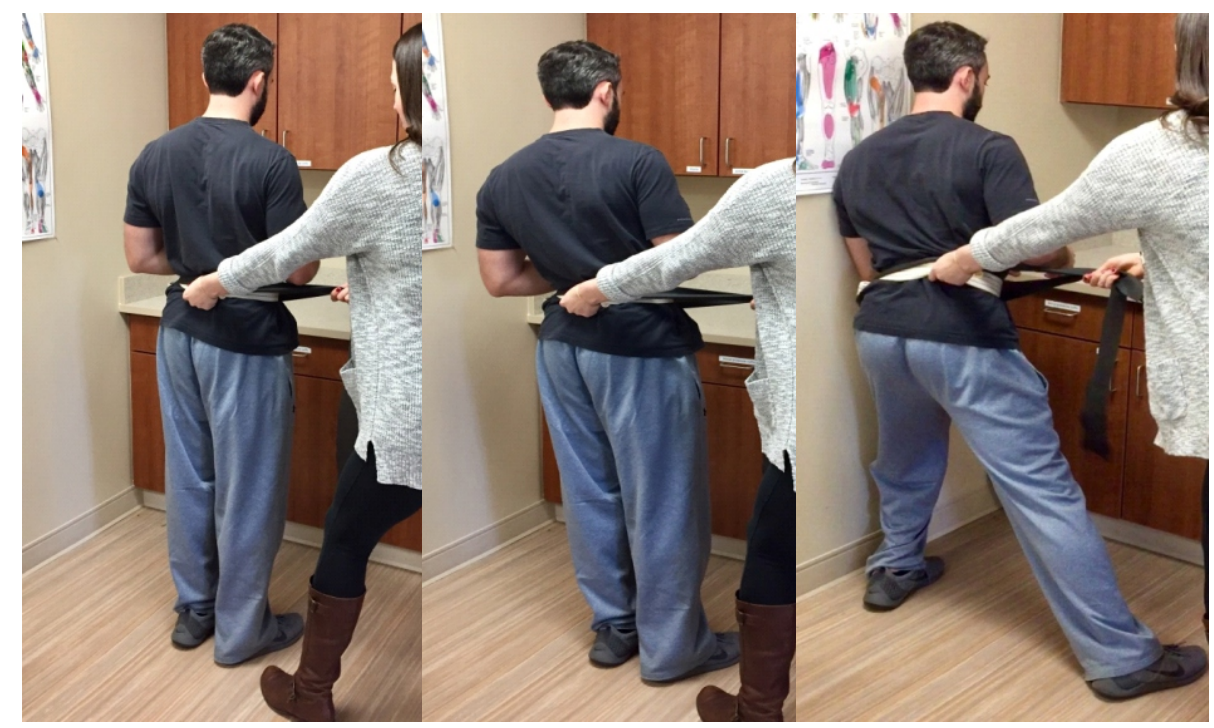

Figure 1. Facilitation of lateral protective step with a resistance band

2. Snow-shoveling simulation (Figure 2). Due to the client's complaints of falling while shoveling snow, a simulation was set up to address reactive balance strategies during this task. The client performed a mini squat with a staggered stance to pick up an 8-pound weighted ball off of a 12" high surface. He then rotated to the side of the posterior foot and threw the ball in a posterolateral direction, similar to his snow-shoveling mechanics. When the 
external weight was displaced, the client had to take a compensatory step to prevent a fall.

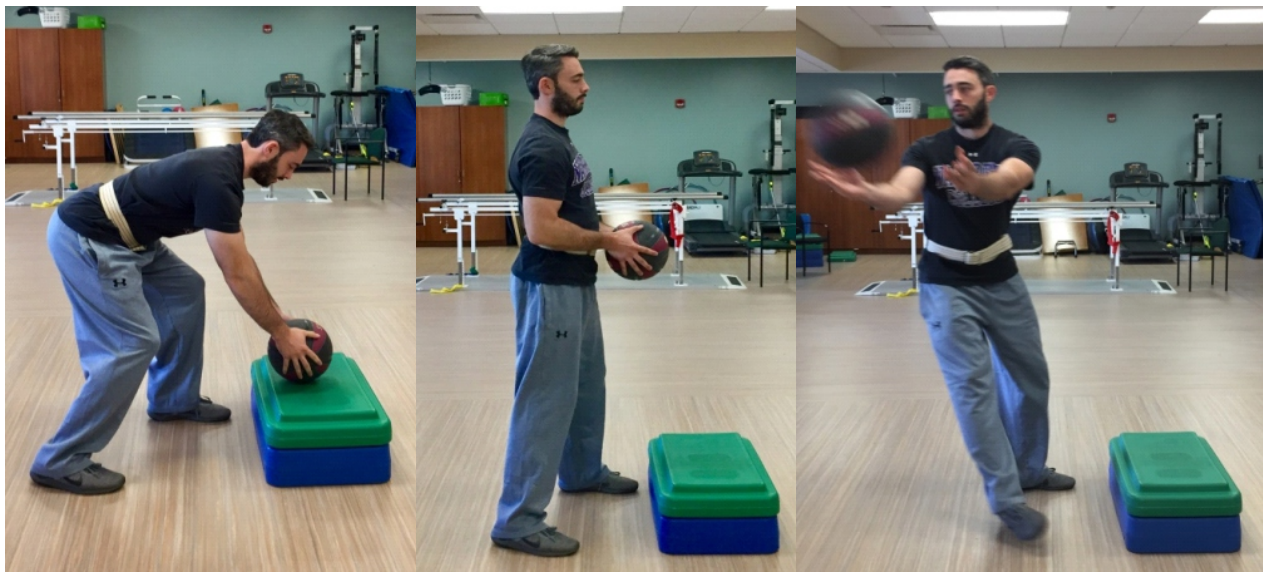

Figure 2.Snow-shoveling simulation

3. Fishing simulation (Figure 3). The client stood on a firm surface and threw a tennis ball connected to a rope to a person sitting on a scooter 15 feet away. He was instructed to wind the rope up as if he were reeling in a fish. The person on the scooter moved from side to side and unexpectedly let go of the rope when 5 feet away so that the client had to take a compensatory step backwards. After demonstrating success with this task, he stood on a tilt board with a curb step behind him to accommodate the backwards step. This activity was performed in a harness system with $15 \%$ body weight support.

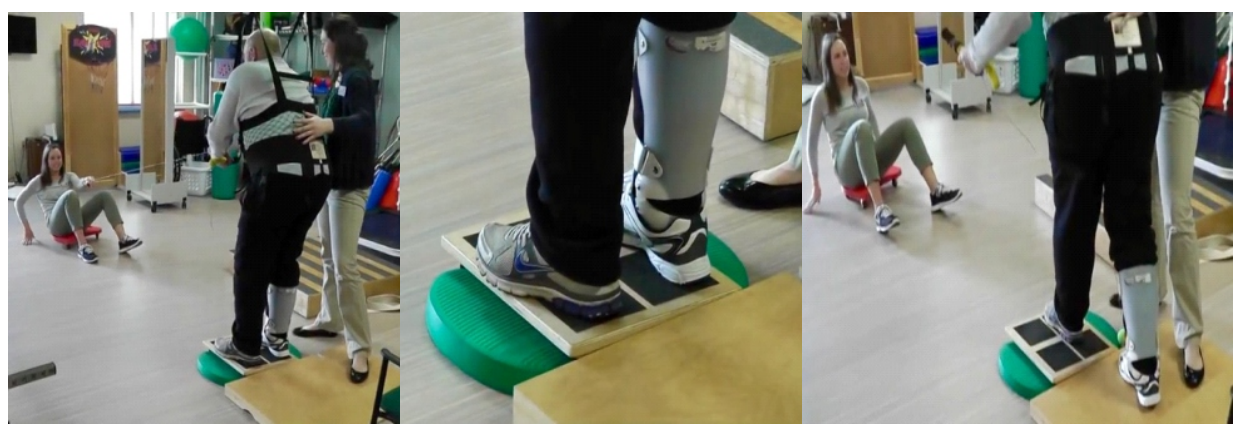

Figure 3.Fishing simulation

Upon initiation of the lateral stepping strategy with the resistance band, the client did not step at all or required 2-3 compensatory steps and progressed to consistently taking 1-2 compensatory steps $100 \%$ of the time by the final session. Once the client was able to demonstrate an effective stepping strategy, he was able to progress to the snow-shoveling simulation. On the first attempt of this activity, the client required assistance to correct a loss of balance $\sim 75 \%$ of the time and progressed to only requiring assistance $\sim 5 \%$ of the time during the final session. At this time, the fishing simulation activity was introduced. Upon first attempt of this activity, he required up to 4 small steps to prevent a fall and progressed to consistently utilizing an appropriate and safe stepping strategy with only 1-2 steps.

\section{Results}

Following 8 sessions of an intervention program focusing on PBT, improvements in balance, fear of falling, lower extremity strength, aerobic capacity, and a decreased number of falls were noted. Outcomes are summarized below in Table 1.

Functional stability during gait improved from scores on the DGI of 15/24 to $17 / 24$, but not enough to reach the minimal detectable change (MDC) for the stroke population (no data available for $\mathrm{SCl}$ population) as determined by Jonsdottir, et al. ${ }^{24}$ Improvements in lower extremity strength were noted as the client's time on the 5TSS decreased from 23.9 to 15.8 seconds, just marginally missing the cut-off score of less than 15 seconds predicting multiple falls in someone with incomplete $\mathrm{SCl}^{31}$ and with no minimal clinically important difference (MCID) or MDC data for SCI. Aerobic capacity improved as demonstrated by a change from 734 to 913 feet in the 6MWT, exceeding the MDC for SCI of 150 feet established by Lam, et al. ${ }^{32}$ Additionally, the client's fear of falling made a drastic improvement from scoring a $43.8 \%$ to 
$73.8 \%$ on the ABC Scale, exceeding the cut-off score of less than $67 \%$ indicating an increased risk for falling in older adults ${ }^{33}$ and with no specific SCl data available. Mobility and balance during gait also made an improvement as noted by a decrease from 17.1 to 12.7 seconds in the TUG, however not reaching the MDC for SCI of 10.8 seconds established by Van Hedel, et al. ${ }^{29}$ Finally, the client reported 2-3 falls per week at the time of initial evaluation and consistently had 0 falls for the final 3 weeks of therapy.

Table 1.Clinical Outcome Measure Results

\begin{tabular}{|c|c|c|}
\hline Outcome Measure & Initial Evaluation & Discharge \\
\hline Dynamic Gait Index & $15 / 24$ & $17 / 24$ \\
\hline $\begin{array}{l}\text { Five Time Sit to Stand } \\
\text { (seconds) }\end{array}$ & 23.9 & 15.8 \\
\hline $\begin{array}{c}\text { Six Minute Walk Test } \\
\text { (feet) }\end{array}$ & 734 & $913^{*}$ \\
\hline $\begin{array}{l}\text { Activities-Specific } \\
\text { Balance Confidence } \\
\text { Scale (percent) }\end{array}$ & 43.8 & $73.8^{*}$ \\
\hline $\begin{array}{c}\text { Timed Up and Go } \\
\text { (seconds) }\end{array}$ & 17.1 & 12.7 \\
\hline Falls Per Week & $2-3$ & $\begin{array}{c}0 \text { (consistent for final } 3 \\
\text { weeks of therapy) }\end{array}$ \\
\hline
\end{tabular}

*Exceeded the established MDC, MCID, or cut-off score values (for SCI when available) from the literature for each outcome measure.

\section{Discussion}

This case report demonstrates that an intervention plan focusing on PBT and retraining reactive balance strategies within functional activities can lead to decreased falls, improved balance confidence, and ability to initiate an effective protective stepping response. In addition, improvements were noted in lower extremity strength and aerobic capacity in a 58year-old male 18 months post incomplete SCI. Interventions did not require sophisticated equipment to complete and instead allowed the client to complete challenging real, functional activities while in a safe and controlled environment.

Results from this study were similar to Mansfield, et al. ${ }^{9}$ finding that compensatory reactive stepping can be retrained in the stroke population and also similar to Jobdges, et al. ${ }^{17}$ in those with Parkinson's. Comparable results were also found to Thigpen,et al. ${ }^{18}$ in which those with an incomplete $\mathrm{SCI}$ showed ability to adapt to repeated perturbations, but results from this case report were able to conclude similar results without the use of sophisticated laboratory equipment.

Although no studies specifically investigated the effects of task-specific PBT in the incomplete $\mathrm{SCl}$ population, improvements in similar outcome measures were seen with various types of training. Foster, et al. ${ }^{34}$ found improvements in balance, balance confidence, and functional mobility measured by the TUG in the incomplete $\mathrm{SCl}$ population, but were a result of performing backward walking training compared to the PBT performed in this case report. DiPiro,et al. ${ }^{35}$ also found a similar improvement in aerobic capacity in those with incomplete $\mathrm{SCl}$ and Hicks, et al. ${ }^{36}$ found the same in a meta-analysis of those with complete injuries, but both results were after performing specific, aerobic training. Harkema, et al. ${ }^{20}$ also found an improvement in aerobic capacity (measured through 6MWT) and balance (measured by Berg Balance Scale), but after receiving task-specific loco-motor and step training.

The client participated in outpatient therapy focusing on general balance training, gait training, and core/hip strengthening for 16 months. Although he did make gradual gains throughout these 16 months, he continued to experience 2-3 falls per week. After eight sessions of PBT performed during task-specific, functional tasks, he made substantial improvements in all standardized outcome measures and decreased his number of falls to zero per week. Balance confidence and falls were expected to increase simply due to the nature of the intervention, but improvements in strength and aerobic capacity measures were unexpected. There is a question of whether it was solely the addition of PBT that improved 
outcomes within a variety of different areas, or the combination of this plus incorporating salient activities into therapy.

Although this case report provides insight into the ability to retrain and improve reactive balance strategies in a client with chronic incomplete $\mathrm{SCl}$, there are several limitations to be considered. First, as noted, the client was attending outpatient physical therapy once weekly in addition to participating in the eight weeks of intervention at the PT program clinic. Although he had been attending this clinic for 16 months prior with gradual improvements in objective measures, he subjectively stated, "Your approach to dealing with my difficulties has opened new doors of treatment that may not have been considered." Second, although several standardized outcome measures were utilized, one specific to reactive balance such as the Mini Balance Evaluation Systems Test (Mini BESTest) ${ }^{37}$ was not performed with this client. Third, terminology regarding PBT or reactive balance training is not consistent throughout the literature, making it difficult to fully encapsulate all of the available evidence on this topic. Finally, due to this study being a case report, there was only one client participating in this intervention plan and similar results cannot be assumed with patients of the same or different diagnoses.

Future research should focus on incorporating facilitation of reactive balance strategies and PBT with task-specific interventions with incomplete $\mathrm{SCl}$ clients as well as investigate the effect of this type of intervention program on standardized outcome measures.

\section{Conclusion}

This case report describes the results of retraining effective reactive balance strategies through the use of PBT in an individual with chronic incomplete SCl. The intervention plan consisted of retraining the protective stepping reaction within salient and task-specific activities, with improvements noted in all functional outcome measures and decreased number of falls. Interventions were designed to be feasible and practical for any clinician to keep the client safe while maintaining adequate challenge to induce change and while also being salient to the patient to maintain motivation level. Results of this case report suggest one potential avenue for intervention for the incomplete SCI population to improve reactive balance abilities and in turn reduce falls, even greater than one-year post injury. 


\section{References}

1. Spinal Cord Injury Facts and Figures at a Glance. National Spinal Cord Injury Statistical Center through University of Alabama at Birmingham website. Retrived from: https://www. nscisc.uab. edu/Public/Facts\%202016.pdf

2. Phonthee S, Saengsuwan J, Siritaratiwat W, et al. Incidence and factors associated with falls in independent ambulatory individuals with spinal cord injury: a 6-month prospective study. Phys Ther. 2013;93(8):1061-1072.

3. Tamburella F, Scivoletto G, Molinari M. Balance training improves static stability and gait in chronic incomplete spinal cord injury subjects: a pilot study. Eur J Phys Rehab Med. 2013;49(3): 353-364

4. Brotherton S, Krause J, Nietert P. Falls in individuals with incomplete spinal cord injury. Spinal Cord. 2007;45(1): 37-40.

5. Horak F, Henry S, Shumway-Cook A. Postural perturbations: new insights for treatment of balance disorders. Phys Ther. 1997;77(5): 517-533.

6. Mansfield A, Wong JS, Bryce J, et al. Does perturbation-based balance training prevent falls? Systematic review and meta-analysis of preliminary randomized controlled trials. Phys Ther. 2015;95(5): 700-709.

7. Salot $P$, Patel $P$, Bhatt $T$. Reactive balance in individuals with chronic stroke: biomechanical factors related to perturbation-induced backward falling. Phys Ther. 2016;96(3): 338-347.

8. Lakhani B, Mansfield A, Inness E, Mcllroy W. Compensatory stepping responses in individuals with stroke: a pilot study. Physiother Theor Pr. 2011;27(4): 299-309.

9. Mansfield A, Inness E, Mcllroy W, et al. Training rapid stepping responses in an individual with stroke. Phys Ther. 2011;91(6): 958-969.

10. Mansfield A, Inness E, Wong J, et al. Is impaired control of reactive stepping related to falls during inpatient stroke rehabilitation? Neurorehab Neural Re. 2013;27(6): 526-533.

11. Yungher D, Morgia J, Rogers $M$, et al. Short-term changes in protective stepping for lateral balance recovery in older adults. Clinical Biomechanics. 2012;27(2): 151-157.

12. Bhatt T, Pai Y. Prevention of slip-related backward balance loss: the effect of session intensity and frequency on long-term retention. Arch Phys Med Rehab. 2009;90(1): 34-42.

13. Pai $Y$, Bhatt $T$, Wang $E$, et al. Inoculation against falls: rapid adaptation by young and older adults to slips during daily activities. Arch Phys Med Rehab. 2010;91(3): 452-459.

14. Maki B, Edmondstone M, Mcllroy W. Age-related differences in laterally directed compensatory stepping behavior. Gerontol A-Biol. 2000;55(5): M270-M277.

15. Mansfield A, Peters A, Liu B, et al. Effect of a perturbation-based balance training program on compensatory stepping and grasping reactions in older adults: a randomized controlled trial. Phys Ther. 2010;90(4): 476-491.

16. Patel $P$, Bhatt $T$. Adaptation to large-magnitude treadmill-based perturbations: improvements in reactive balance response. Physiol Rep. 2015;3(2): e12247.

17. Jöbges M, Heuschkel G, Pretzel C, et al. Repetitive training of compensatory steps: a therapeutic approach for postural instability in Parkinson's disease. J Neurol Neurosur Ps. 2004;75(12): $1682-1687$.

18. Thigpen M, Cauraugh J, Behrman A, et al. Adaptation of postural responses during different standing perturbation conditions in individuals with incomplete spinal cord injury. Gait Posture. 2009;29(1): 113-118.

19. Lemay J, Duclos C, Nadeau S, et al. Postural and dynamic balance while walking in adults with incomplete spinal cord injury. J Electromyogr Kines. 2014;24(5): 739-746.

20. Harkema S, Schmidt-Read M, Lorenz D, et al. Balance and ambulation improvements in individuals with chronic incomplete spinal cord injury using loco-motor training-based rehabilitation. Arch Phys Med Rehab. 2012;93(9): 1508-1517.

21. Jones M, Evans N, Morrison S, et al. Activity-based therapy for recovery of walking in chronic spinal cord injury: results from a secondary analysis to determine responsiveness to therapy. Arch Phys Med Rehab. 2014;95(12): 2247-2252.

22. Behrman A, Bowden M, Nair P. Neuroplasticity after spinal cord injury and training: an emerging 
paradigm shift in rehabilitation and walking recovery. Phys Ther 2006;86(10): 1406-1425.

23. Sibley K, Brooks D, Jaglal S, et al. Development of a Theory-Based Intervention to Increase Clinical Measurement of Reactive Balance in Adults at Risk of Falls. J Neurol Phys Ther. 2016;40(2): 100-106.

24. Jonsdottir J, Cattaneo D. Reliability and validity of the dynamic gait index in persons with chronic stroke. Arch Phys Med Rehab. 2007;88(11): 1410-1415.

25. Bohannon R. Reference values for the five-repetition sit-to-stand test: a descriptive meta-analysis of data from elders. Perceptual And Motor Skills. 2006;103(1): 215-222.

26. Butland R, Pang J, Gross E, et al. Two-, six-, and 12-minute walking tests in respiratory disease. $B R$ Med J (Clin Red Ed). 1982;284(6329): 1607-1608.

27. American Thoracic Society. ATS Statement: Guidelines for the Six-Minute Walk Test. Am J Resp Crit Care Med. 2002; 166: 111-117.

28. Powell LE, Myers AM. The Activities-specific Balance Confidence (ABC) scale. J Gerontol ABiol;50A(1): M28-M34.

29. Van Hedel H, Wirz M, Dietz V. Assessing walking ability in subjects with spinal cord injury: validity and reliability of 3 walking tests. Arch Phys Med Rehab. 2005;86(2): 190-196.

30. Podsiadlo D, Richardson S. The timed "Up \& Go": a test of basic functional mobility for frail elderly persons. J Am Geriatr Soc. 1991;39(2):142-148.

31. Srisim K, Saengsuwan J, Amatachaya S. Functional assessments for predicting a risk of multiple falls in independent ambulatory patients with spinal cord injury. J Spinal Cord Med. 2015;38(4): 439-445.

32. Lam T, Noonan V, Eng J. A systematic review of functional ambulation outcome measures in spinal cord injury. Spinal Cord. 2008;46(4): 246-254.

33. Lajoie Y, Gallagher S. Predicting falls within the elderly community: comparison of postural sway, reaction time, the Berg balance scale and the Activities-specific Balance Confidence (ABC) scale for comparing fallers and non-fallers. Arch Gerontol Geriat. 2004;38(1): 11-26.

34. Foster H, DeMark L, Spigel P, et al. The effects of backward walking training on balance and mobility in an individual with chronic incomplete spinal cord injury: A case report. Physiother Theor Pr. 2016;32(7): 536-545.

35. DiPiro N, Embry A, Fritz S, et al. Effects of aerobic exercise training on fitness and walking-related outcomes in ambulatory individuals with chronic incomplete spinal cord injury. Spinal Cord. 2016;54(9): 675-681.

36. Hicks A, Martin Ginis K, Pelletier C, et al. The effects of exercise training on physical capacity, strength, body composition and functional performance among adults with spinal cord injury: a systematic review. Spinal Cord. 2011;49(11): 1103-1127.

37. Franchignoni F, Horak F, Godi M, et al. Using psychometric techniques to improve the balance evaluation systems test: the mini-bestest.J Rehabil Med. 2010;42(4):323-331. 